\title{
Les élèves issus de l'immigration sont-ils des élèves à besoins éducatifs particuliers?
}

Auteure(s)

Stéphanie Bauer, Haute École Pédagogique du Canton de Vaud, Suisse, stephanie.bauer@hepl.ch

Corina Borri-Anadon, Université du Québec à Trois-Rivières, Canada, corina.borri-anadon@uqtr.ca

Moira Laffranchini Ngoenha, Haute École Pédagogique du Canton de Vaud, Suisse,

moira.laffranchini-ngoenha@hepl.ch 


\section{REVUE HYBRIDE DE L'ÉDUCATION}

\section{Résumé}

La recherche en inclusion scolaire s'est intéressée principalement aux élèves porteurs de handicap et peu aux élèves issus de l'immigration. Notre contribution porte sur les promesses et défis de la reconnaissance des rapports ethniques au sein de l'inclusion scolaire. Nous mobilisons à cet effet la notion d'élèves à besoins éducatifs particuliers en examinant de manière critique les enjeux conceptuels et pratiques qu'elle soulève, notamment lorsqu'elle est utilisée pour désigner les élèves issus de l'immigration. Seront également dégagées des pistes de réflexion pour conduire l'école à une mobilisation des ressources éducatives particulières amenées par ces mêmes élèves.

Mots-clés: inclusion scolaire, élèves issus de l'immigration, élèves à besoins éducatifs particuliers, élèves à ressources éducatives particulières 


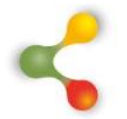

\section{REVUE HYBRIDE DE L'ÉDUCATION}

\section{Introduction}

Les politiques d'éducation inclusive sont aujourd'hui à l'agenda de bon nombre de systèmes éducatifs occidentaux. Initiée en grande partie par I'UNESCO, la réflexion sur l'ouverture de l'école à tous les élèves a donné lieu à une série de recommandations nationales et internationales, se transformant parfois localement en politiques éducatives concrètes. Un double objectif était à la base de ces réformes : la conception globale d'une école plus ouverte et plus juste ainsi que la scolarisation des élèves handicapés dans l'école ordinaire (Ramel, 2015). L'école inclusive, favorisant la réussite de tous serait une réponse à cette double demande. Parallèlement, l'école publique s'est également posé la question de la réussite scolaire des élèves issus de l'immigration ${ }^{1}$ et propose aujourd'hui une série de mesures pour faciliter leur intégration scolaire, allant des structures d'accueil à l'apprentissage du français (Akkari, 2009).

L'objectif de cet article est d'interroger la place des élèves issus de l'immigration dans le paradigme inclusif, en se demandant plus précisément s'ils peuvent être considérés comme des élèves à besoins éducatifs particuliers et quels seraient les enjeux d'une telle acception. À la suite de Gremion, Noël et Ogay (2013), qui formulent la question suivante : "Une pédagogie inclusive serait-elle à même de constituer un point d'alliance de l'éducation interculturelle et de la pédagogie spécialisée ? " (p.64), nous mettrons en évidence les points de convergence et de divergences entre ces approches. Par la suite, nous interrogerons également les différentes conceptions de la notion de besoins éducatifs particuliers, leurs dérives, et les questions que ces dernières soulèvent pour les élèves issus de l'immigration. Enfin, nous proposerons un renversement terminologique afin de mettre à jour les élèves issus de l'immigration comme des élèves à ressources particulières dans une école à besoins particuliers et soulignerons quelques implications pour la pratique pédagogique et la formation du personnel scolaire.

\section{Quelle place pour les élèves issus de l'immigration au sein du paradigme inclusif ?}

Le paradigme ${ }^{2}$ de l'école inclusive qui vise "l'école pour tous " s'est développé à partir de l'influence de différent-e-s agent-e-s. L'UNESCO

\footnotetext{
${ }^{1}$ Bien que le sens de cette expression varie selon les contextes, nous retenons ici la définition de l'OCDE (2016), soit les élèves qui sont, ou dont les deux parents sont, nés dans un pays étranger.

2 Nous regroupons sous le vocable «paradigme inclusif» un ensemble de fondements philosophiques et moraux, sociologiques et juridiques, ainsi que
} 


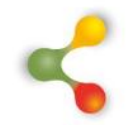

\section{REVUE HYBRIDE DE L'ÉDUCATION}

notamment a joué un rôle clé en promouvant la réforme des systèmes éducatifs en faveur d'une scolarisation des élèves marginalisés dans le système ordinaire (Artiles et Dyson, 2005 ; Ramel et Vienneau, 2016). Le mouvement de l'inclusion scolaire est devenu international. Les principes directeurs pour l'inclusion dans l'éducation (UNESCO, 2009) affirment la nécessité d'une éducation de qualité pour tous les élèves par le biais de la pédagogie inclusive, " seule garante d'une participation pleine et entière à la vie scolaire et sociale » (Ramel et Vienneau, 2016, p.34).

Le regard porté sur la différence a donc évolué suite aux critiques des mesures séparatives, jugées peu efficaces, et à la normativité de l'école face aux élèves porteurs de ces « différences » (Perregaux, 2010). Plutôt que de parler des élèves handicapés, dans une approche uniquement psychomédicale où le trouble est vu comme intrinsèque à la personne et la définit dans sa totalité, on préfère parler d'élèves " porteurs de handicaps " ou " en situation de handicap ", afin de souligner le caractère contextuel du trouble en question (Barral et Roussel, 2002). Cette approche situationnelle (Fougeyrollas, 2002) met l'accent sur la production sociale du handicap et la manière dont une caractéristique peut être perçue comme handicap, en fonction de la situation donnée.

Traditionnellement, la situation spécifique des élèves issus de l'immigration a été l'objet particulier des approches interculturelles. Sur les principes généraux, ces dernières témoignent d'une évolution similaire à celle qu'a connue le paradigme inclusif, influencé également par les organisations internationales comme l'UNESCO et le Conseil de l'Europe (Akkari, 2009). Tout comme le paradigme inclusif, elles remettent en question les mesures de scolarisation séparatives à l'égard des élèves issus de l'immigration, dominantes jusque dans les années 80 (Gremion, Noël et Ogay, 2013). Ainsi, sur le plan des principes fondateurs, ces différentes approches convergent vers des objectifs communs d'équité et de justice sociale.

L'école inclusive se veut en effet une rupture face à la scolarisation séparée de certains publics. C'est une école pour tous les élèves, quel que soit leur besoin. Ce changement de perspective témoigne de la transformation du regard que pose l'école sur ces élèves présentant des caractéristiques identifiées comme moins conformes aux attentes scolaires. L'adaptation des pratiques pédagogiques a participé à cette réflexion, en se basant notamment sur le constat plus général, amené par la sociologie de l'éducation, de la faillite du modèle de l'égalité des chances et de la méritocratie (Dubet, 2009). Ces recherches montrent en effet l'échec de

pédagogiques et psychopédagogiques (voir notamment Prud'homme, Duchesne, Bonvin et Vienneau, 2016 et Potvin, 2013), qui vise «la transformation de l'école pour lui permettre de répondre à la diversité des besoins éducatifs de tous les élèves » (Prud'homme et Ramel, 2016, p.16). 


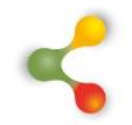

\section{REVUE HYBRIDE DE L'ÉDUCATION}

l'école à faire réussir tous les publics, indépendamment de leurs caractéristiques individuelles. Par exemple, selon les enquêtes PISA (OCDE, 2016), certains pays présentent des disparités de résultats significatifs entre les groupes d'élèves selon leur origine sociale, migratoire ou encore leur sexe.

En conséquence, ce sont les valeurs de justice et d'équité qui nécessitent d'être repensées puisque l'école juste ne peut plus être l'école qui traite tous les élèves de la même manière. Selon Larochelle-Audet et al. (2018), ces deux notions, justice et équité, sont interreliées à travers les canaux de redistribution et de reconnaissance. L'école inclusive porte au cœur de sa réflexion la capacité du système à faire réussir tous les élèves. La notion de justice n'est donc plus pensée comme une manière de traiter les élèves de la même manière, mais comme une distribution équitable des ressources et une reconnaissance par laquelle les caractéristiques des élèves, leurs différences inter et intra-individuelles, deviennent légitimes à l'école, permettant ainsi de réduire les inégalités et d'assurer une réussite partagée par le plus grand nombre. Les valeurs de justice sociale et d'équité sont donc au cœur des fondements du paradigme inclusif (Prud'Homme, Duchesne, Bonvin et Vienneau, 2016).

Néanmoins, la légitimité de la place des élèves issus de l'immigration dans l'école inclusive ne va pas forcément de soi. D'une part, Ramel et Vienneau (2016) rappellent que les déclarations et conventions internationales ayant contribué à l'avènement du paradigme inclusif ont "contribué à la formation de nos représentations de l'éducation inclusive en se focalisant davantage sur les enfants en situation de handicap ou en très grande difficulté »(p.25). Bien que depuis 1990 on assiste à l'avènement des discours politiques misant sur l'éducation pour tous, force est de constater que la diversité des élèves est souvent réduite aux élèves en situation de handicap (Benoit et Plaisance, 2009).

D'autre part, comme le relèvent certaines auteurs (Gremion, Noël et Ogay, 2013 ; Mc Andrew et al., 2015 ; Perregaux, 2010), des représentations assez divergentes existent auprès du personnel éducatif quant à la valeur sociale attribuée au statut de la différence centrée sur des marqueurs culturels ou linguistiques. Perregaux (2010), puis Gremion, Noël et Ogay (2013) montrent en effet que le discours social à propos de la différence reconnait plus légitime la présence du handicap en classe ordinaire que celui de la différence culturelle : " II semble que la société accepte mieux l'idée de devoir donner une place aux personnes nées ici avec une déficience, plutôt qu'aux personnes sans handicap nées ailleurs, quand cet ailleurs semble culturellement trop éloigné » (Perregaux, 2010, p.63). La question de l'immigration est une question éminemment sensible, voire politique, sa pertinence et sa contribution sociale étant toujours débattues dans l'espace public. 


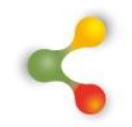

\section{REVUE HYBRIDE DE L'ÉDUCATION}

Enfin, l'intégration des élèves issus de l'immigration dans le paradigme inclusif soulève un risque quant à une dilution des questions spécifiques dans un discours général sur la "diversité » (Gremion, Noël et Ogay, 2013). À des questions particulières, en termes d'enjeux pédagogiques, politiques, scientifiques, est apportée une réponse universelle, voire unique : la prise en compte des besoins éducatifs particuliers.

Ainsi, au cœur des débats sur l'inclusion s'expriment parfois des tensions entre d'une part, une reconnaissance spécifique des enjeux particuliers de certains groupes d'élèves et d'autre part, une réponse générale, à travers laquelle ces enjeux disparaissent dans la vaste notion de besoins éducatifs particuliers. Si les populations spécifiques, qui ont fait l'objet de la réflexion sur l'inclusion scolaire et de la recherche sur la question, renvoyaient surtout aux élèves porteurs de handicaps (Lindsay, 2007), l'objectif inclusif de la réussite pour tous permet d'élargir la question à tous les profils d'élèves. En effet, il ne s'agit plus de penser une école pour certains, mais de penser une école pour tous où chaque élève, avec ses spécificités, pourrait apprendre et progresser.

\section{La notion de besoins éducatifs particuliers}

L'avènement de l'approche situationnelle quant au handicap (Fougeyrollas, 2002) a permis l'émergence de nouvelles façons d'aborder les difficultés des élèves dans le champ de l'adaptation scolaire, en introduisant notamment la notion de besoins éducatifs particuliers (Pelgrims, 2012). Selon Plaisance (2000), l'origine de cette notion remonte au contexte britannique de la fin des années 70 où différentes politiques éducatives ont cherché à se distancier des mesures ségrégatives de l'éducation spéciale. Depuis, elle a été l'objet de fortes critiques, liées notamment à la perspective intégrative dans laquelle cette notion a vu le jour, c'est-à-dire " une perspective d'action qui prolonge, certes en les améliorant, les politiques d'éducation spéciale, mais qui peut se limiter à la présence physique d'une enfant 'à problème' dans un contexte ordinaire " (Plaisance, 2000, p.26). Malgré ces critiques, la notion de «besoin » occupe encore aujourd'hui une place centrale dans les injonctions internationales et politiques nationales s'inscrivant dans le paradigme inclusif (UNESCO, 2009). En effet, en reposant sur une critique de la perspective psychomédicale, cette notion sous-entend que les dimensions sociales et culturelles de l'élève doivent être considérées dans la compréhension de la situation de ce dernier. Elle permet également de dissocier diagnostic et traitement, c'est-à-dire que la nature des difficultés n'impose plus à elle seule le type de soutien à offrir à l'élève. En d'autres termes, on passe d'une différenciation structurale à une différenciation pédagogique (Lavoie, Thomazet, Feuilladieu, Pelgrims et Ebersold, 2013). Ce faisant, le rôle de l'école dans l'identification de ces besoins éducatifs 


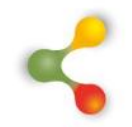

\section{REVUE HYBRIDE DE L'ÉDUCATION}

particuliers est interrogé, puisque ces derniers dépendent de la capacité de l'école même à adapter le curriculum, l'enseignement, l'organisation et les ressources afin de favoriser l'apprentissage des élèves (UNESCO, 1997). Bien que le changement terminologique du handicap vers le besoin soit considéré comme un pas dans la bonne direction, « une étape pertinente pour aider à penser puis agir autrement »(Thomazet, 2012, p.16), il apparaît que cette notion demeure imprécise, variable d'un contexte à l'autre et peu définie (Ebersold et Detraux, 2013). II convient donc de retracer ici les diverses conceptions de la notion de besoins éducatifs particuliers et leurs implications sur les élèves issus de l'immigration.

Une première conception de la notion d'élèves à besoins particuliers s'appuie sur sa correspondance avec celle de déficience. Ici, la notion est utilisée pour " désigner des individus, pour qualifier ceux et celles, des personnes, des élèves qui présentent un handicap, une déficience " (Pelgrims, 2012, p.8). Dans ce sens, elle repose sur l'identification, par le recours à des professionnel-le-s spécialisé-e-s, de ces besoins définis à partir de la nature des difficultés rencontrées par l'élève. C'est en quelque sorte la difficulté qui définit le besoin. S'inscrivant toujours dans le sillon de la perspective psychomédicale, cette conception ne permet pas d'en dépasser les critiques, tant dans la (non) reconnaissance des dimensions sociales et culturelles constitutives de la situation de l'élève que dans le soutien qui en découle (Ebersold et Detraux, 2013 ; Pelgrims, 2012).

Dans le cas des élèves issus de l'immigration, une telle conception tend, d'une part, à occulter leurs réalités et expériences spécifiques ou encore à en atténuer les possibles effets, et d'autre part, à considérer ces réalités et expériences à partir d'une perspective déficitariste ${ }^{3}$, c'est-à-dire en leur attribuant un effet amplificateur, voire catalyseur, des difficultés scolaires (Borri-Anadon, Savoie-Zajc et Lebrun, 2015). Ainsi, par sa centration sur l'individu et ses déficits, une telle conception de la notion d'élèves à besoins éducatifs particuliers renforce les catégories diagnostiques utilisées à l'égard des élèves en difficulté et, par le fait même, est susceptible de contribuer à une suridentification de certains sous-groupes d'élèves issus de l'immigration dans des populations dites handicapées (Borri-Anadon, 2016). L'élève issu de l'immigration est alors identifié comme porteur de difficultés intrinsèques alors que ces dernières peuvent être liées à ses réalités et expériences sur les plans linguistique, scolaire et social et/ou au regard que les intervenants portent sur celles-ci. Par ses conséquences sur

\footnotetext{
${ }^{3}$ La perspective déficitariste conçoit les difficultés scolaires comme provenant des particularités (autrefois organiques et aujourd'hui plus d'ordre psychosocial) d'un groupe culturel, en tant que " lacunes » (Borri-Anadon, 2014 ; Gauthier, 2005). II s'agit donc d'expliquer les difficultés des élèves ou de justifier les interventions à leur égard à travers des " pédagogies du manque et de la compensation " (Terrail, 2016, p.152).
} 


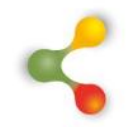

\section{REVUE HYBRIDE DE L'ÉDUCATION}

l'élève lui-même, notamment au plan identitaire et de son engagement scolaire ainsi que sur les représentations et attentes des intervenants scolaires à son égard, la suridentification engendre une stigmatisation, qu'elle soit intériorisée et/ou attribuée. En outre, le classement scolaire spécialisé, susceptible de découler de la suridentification, contribue à la persistance de logiques de ségrégation pour certaines minorités racisées au sein de l'espace scolaire (Artiles, Trent, et Palmer, 2004 ; Cartledge, 2005 ; Hibel et Jasper, 2012 ; Waitoller, Artiles et Cheney, 2009).

Une seconde conception de la notion de besoins éducatifs particuliers repose plutôt sur la réponse offerte à ces besoins. À cet égard, les élèves à besoins éducatifs particuliers seraient ceux pour lesquels un soutien additionnel est jugé nécessaire pour favoriser leur réussite. C'est notamment le choix de l'OCDE (2008) qui propose la définition suivante : "les personnes ayant des besoins éducatifs particuliers se définissent par les ressources publiques ou privées supplémentaires engagées pour le financement de leur éducation» (pp.21-22). S'inscrivant dans une "approche consommatoire », qui réduit l'activité pédagogique à une «prestation » et le besoin à un « service » (Ebersold et Detraux, 2013, p.112), cette conception des besoins éducatifs particuliers aurait pour effet de limiter le rôle des systèmes éducatifs à l'allocation de ressources, sans questionner la qualité du soutien offert ou les dynamiques systémiques pouvant participer à l'émergence de ces besoins. D'autre part, cette conception aurait également comme conséquence d'élargir le spectre des élèves à besoins éducatifs particuliers à tout élève reconnu comme étant vulnérable, ce " qui a renforcé la médicalisation de l'échec scolaire en transformant tout élève en élève potentiellement à risque " (Ebersold et Detraux, 2013 p.112). Cette notion du besoin, si polysémique qu'elle pourrait correspondre à tous les élèves, illustre les limites de cette approche par le service.

Cette acception peut être illustrée par le cas des élèves non-locuteurs de la langue de scolarisation, qui sont désignés à partir des services de soutien à l'apprentissage du français. Dans ce sens, la réponse à la question de la définition des besoins éducatifs particuliers se fait par la mesure plutôt que par l'identification même de ces besoins, les restreignant ainsi aux services offerts. Ainsi, certains élèves issus de l'immigration deviennent des élèves non francophones ayant besoin de soutien à l'apprentissage du français. Mais ces élèves ont-ils seulement besoin d'un soutien en français ? Qu'en est-il de la reconnaissance de leurs réalités et expériences reposant sur un marqueur culturel et non linguistique et de la légitimité donnée à l'expression de ces dernières ? Et quid des élèves de $2^{\mathrm{e}}$ génération ou de minorités racisées qui maitrisent la langue d'enseignement, mais qui peuvent avoir besoin de soutien à d'autres égards ? La conception dite " consommatoire » par la réponse aux besoins laisse donc certaines questions ouvertes. 


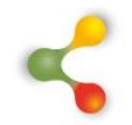

\section{REVUE HYBRIDE DE L'ÉDUCATION}

En outre, cette seconde conception, comme la première, peut aussi être traversée par une perspective déficitariste. En considérant, par exemple, tout élève recevant un soutien pour l'apprentissage de la langue d'enseignement comme un élève à besoins éducatifs particuliers, elle se centre sur ce que l'élève ne sait pas, sans reconnaître qu'il possède un répertoire linguistique, souvent enrichi par ses expériences antérieures, dont celles liées à son statut minoritaire, qui peut être reconnu, valorisé et représenter un apport pour la classe. Ce n'est que le manque à combler qui est perçu, et non pas la richesse de ce qu'il pourrait amener, un constat souvent observé dans l'analyse des représentations des acteurs-trices scolaires (Akkari, Loomis et Bauer, 2012 ; Bauer, 2017 ; Nicollin et Müller Mirza, 2013). Dans ce sens, l'approche consommatoire risque de renforcer les attitudes assimilationnistes, voire misérabilistes, des intervenants à l'égard des élèves issus de l'immigration, participant ainsi au potentiel discriminatoire de leur expérience scolaire (Armand, 2016).

Au regard de ces deux conceptions, la notion d'élève à besoins éducatifs particuliers ne semble pas tenir ses promesses. En effet, qu'elle s'inscrive dans une conception centrée sur la déficience ou sur la réponse offerte, la notion de besoins éducatifs particuliers perpétue une vision individualisante et elle contribue toujours à renforcer la catégorisation des élèves, notamment ceux issus de l'immigration ainsi que leur normalisation au sein d'un espace scolaire qui a des attentes tant sur le plan académique que socioculturel. Ce faisant, elle individualise la réussite ou l'échec et ne permet pas de reconnaître les inégalités qui participent à la construction des difficultés. Comme l'avancent Lavoie et al. (2013) : « à l'instar des catégories médicales, elle est aussi en soi un concept social de classement des élèves qui, à ce titre, peut contribuer à leur discrimination ainsi qu'à leur exclusion » (p.95). Dans ce sens, il semble qu'un écart apparaît entre les visées inclusives de cette notion et sa mise en œuvre dans les pratiques scolaires.

\section{Les élèves issus de l'immigration; des élèves à ressources particulières dans une école à besoins particuliers}

À la lumière des dérives associées à la notion d'élève à besoins éducatifs particuliers, il semble opportun de changer le regard porté sur cette notion. En effet, la mise en avant du besoin, dans sa dénomination comme dans sa réponse par la mesure, n'est-elle pas une autre façon de mettre l'accent sur les prétendus déficits de l'élève sans pour autant valoriser les ressources ? Or, c'est bien la critique de la pédagogie compensatoire et de la vision de la différence comme un écart, qui anime les approches interculturelles et inclusives (Gremion, Noël et Ogay, 2013). Les élèves issus de l'immigration deviennent alors des élèves à besoins éducatifs particuliers parce que l'école ne sait pas rendre possible la prise en compte 


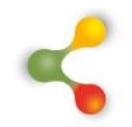

\section{REVUE HYBRIDE DE L'ÉDUCATION}

de leur richesse culturelle et linguistique, des ressources dont ils disposent pour apprendre et pour contribuer à la classe, à l'école et à la société.

Ainsi, nous suggérons un renversement de perspective : les élèves issus de l'immigration ne seraient-ils pas des élèves à ressources particulières dans une école à besoins particuliers ? Ce renversement de perspective rend possible selon nous l'opérationnalisation des trois pistes d'action de la différenciation pédagogique telles que proposées par Prud'homme, Bergeron et Borri-Anadon (2016), soit de reconnaître, de valoriser et de tirer parti de la diversité. Alors que la première implique notamment de réfléchir au caractère dynamique et polyvocal de la diversité, la seconde met de l'avant l'importance d'en soutenir l'expression et la troisième invite à revoir la conception même de l'apprentissage pour que la diversité enrichisse les pratiques d'enseignement (Prud'homme, Bergeron et BorriAnadon, 2016). En effet, comme affirment ces auteurs « différencier, c'est reconnaître fondamentalement que l'équité à l'école repose sur la légitimité d'une diversité de ressources pour apprendre et résoudre une variété de problèmes » (p.171).

Quelles seraient donc les ressources particulières des élèves issus de l'immigration ? II s'agit de ressources permettant à ces élèves d'entrer plus facilement dans les nouveaux apprentissages, mais aussi des ressources permettant à toute la classe de bénéficier de leur participation. L'enjeu réside en effet dans le dépassement des seules reconnaissance et valorisation de la diversité pour aller vers une mobilisation de cette dernière, en tant que ressource pour les élèves eux-mêmes, pour la classe et pour la société. II nous semble au préalable indispensable de souligner que les élèves issus de l'immigration ont des ressources particulières au-delà de leur reconnaissance par le système éducatif. Dans cette lignée, à travers ses exigences et ses attentes, l'école peut risquer d'essentialiser ces ressources des élèves issus de l'immigration, ce qui serait contraire à l'idée de tirer parti de la diversité. C'est pourquoi Armand, Hardy et Lemay (2014) suggèrent le principe de "proposer, mais ne pas imposer » (p.24). Dans ce sens, nous nous concentrons ici sur certains exemples de ressources particulières que les élèves issus de l'immigration sont susceptibles d'apporter avec eux et sur la manière d'en tirer parti en situation d'enseignement-apprentissage.

Un exemple assez immédiat de ressource de certains élèves issus de l'immigration est le plurilinguisime. Or, paradoxalement, il est fréquemment jugé comme une entrave à l'apprentissage (Armand, 2016). Pourtant, le plurilinguisme est considéré aujourd'hui comme une compétence indispensable pour l'employabilité dans un contexte de mondialisation de l'économie : preuve en est l'introduction précoce de l'étude des langues étrangères dans les systèmes éducatifs de nombreux pays au travers le monde (Castellotti, Coste et Duverger, 2008). Quand bien même le 


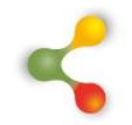

\section{REVUE HYBRIDE DE L'ÉDUCATION}

plurilinguisme est reconnu par les neurosciences comme un facilitateur pour l'apprentissage d'autres langues et pour l'apprentissage tout court, il doit encore affronter les préjugés du statut des langues :: les langues de l'immigration ne revêtent que rarement un statut et un imaginaire positifs et sont très peu présentes officiellement dans le curriculum scolaire (Castellotti et Moore, 2002 ;Cummins, 2001).

Le plurilinguisme est donc un avantage pour l'élève, car il permet d'acquérir de nouvelles compétences métacognitives, en langue, mais également dans d'autres disciplines scolaires (Cummins, 2000). Raisonnablement, la mobilisation des ressources particulières des élèves impliquerait de s'appuyer sur ce savoir de l'élève pour construire le nouveau savoir à acquérir, mais également de le partager avec le groupe classe. Le plurilinguisme de la classe est effectivement un atout pour favoriser l'ouverture à la diversité chez l'ensemble des élèves et réduire du même coup la discrimination basée sur des motifs linguistiques ${ }^{4}$, à condition, comme évoqué ci-haut, " de ne pas imposer une identité linguistique unique à un jeune allophone, fondée sur sa seule langue d'origine au détriment de la reconnaissance d'une identité multiple fondée, entre autres, sur une compétence plurilingue, incluant le français " (ELODIL et ERIT, 2013, p.24).

Un autre exemple de ressource des élèves issus de l'immigration, traversé lui aussi par différents défis de reconnaissance, est celui de la diversité religieuse. À cet égard, Hirsch (2018), qui s'intéresse au cours d'éthique et de culture religieuse implanté au Québec, mentionne que les enseignants devraient être en mesure de " faire appel à des pratiques pédagogiques permettant l'expression de la diversité dans la classe de manière respectueuse et productive » (p.148). En s'intéressant aux différentes manifestations du religieux dans l'environnement des élèves en tant que sujet sensible (Hirsch, Audet et Turcotte, 2015), la diversité religieuse au sein de la classe devient alors l'occasion de nouvelles connaissances, mais aussi d'une négociation, en favorisant chez les élèves le développement de « leur habileté à débattre de leurs points de vue dans l'acceptation des autres possibles » (p.143). La mobilisation de la diversité religieuse des élèves permet ici de pouvoir faire des liens plus concrets avec le vécu religieux, contribuant ainsi à un apprentissage expérientiel plus efficace (Côté, 1998).

Ces ressources amenées par les élèves ont en commun le fait que, si reconnues, valorisées et mobilisées, elles deviennent un outil pédagogique (Prud'homme, Vienneau, Ramel et Rousseau, 2011). Dans ce sens, elles

\footnotetext{
${ }^{4}$ Plusieurs activités d'éveil aux langues à destination des enseignant-e-s existent comme ELODIL (http://www.elodil.umontreal.ca/) ou EOLE (http://eole.irdp.ch/eole/).
} 


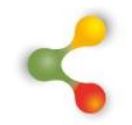

\section{REVUE HYBRIDE DE L'ÉDUCATION}

permettent d'une part à l'élève d'entrer dans les apprentissages et d'être reconnu dans son identité et, d'autre part, à l'enseignant-e de valoriser le savoir de ce dernier et d'en faire bénéficier toute la classe. Tirer parti de la diversité sous-entend donc d'accepter de pouvoir apprendre de celle-ci et également de changer ou d'élargir sa conception de l'apprentissage et de l'enseignement. Ainsi, l'intégration et la réussite scolaire des élèves issus de l'immigration supposent une approche globale que nous inscrivons dans le paradigme de l'école inclusive, comme indiqué dans la première partie de cet article, qui ne se limite pas uniquement à la dimension scolaire. À ce propos, Borri-Anadon, Potvin et Larochelle-Audet (2015) soulignent que

Cette finalité [la finalité de transformation de l'école inclusive] implique d'abord de permettre à toutes et tous de profiter de la richesse de cette diversité en leur offrant des occasions d'être confrontés à des réalités et expériences différentes de la leur. II s'agit d'intéressantes opportunités de développer un regard critique sur les marqueurs de la différence et leur incidence sur le vécu de la discrimination. (p.59)

Abdallah-Pretceille stipulait déjà en 1999 qu'il fallait apprendre la diversité par la diversité et de préférence depuis le plus jeune âge afin qu'elle devienne une expérience positive pour tous les acteurs-trices en jeu dans la relation interculturelle. Cette finalité est également soutenue par I'UNESCO (2013) qui avance que, dans des sociétés de plus en plus hétérogènes, marquées par la mobilité internationale, l'école devrait permettre à tout élève, futur citoyen, de développer des compétences interculturelles qui favorisent le vivre-ensemble et la cohésion sociale.

\section{La formation du personnel scolaire : un enjeu essentiel}

À partir des éléments énoncés plus haut, nous identifions un enjeu essentiel, la formation des professionnel-le-s de l'éducation, afin de pouvoir tirer parti de la diversité culturelle et de dépasser la question de la catégorisation des élèves issus de l'immigration en tant qu'élèves à besoins éducatifs particuliers.

II semble que les représentations du personnel scolaire à l'égard des élèves issus de l'immigration soient toujours marquées par les déficits des élèves. Par exemple, dans le cas québécois, Mc Andrew et al. (2015) soulignent à cet égard " la tendance de nombre d'intervenants scolaires à expliquer les problèmes vécus par certains élèves par divers types de déficits sous-estimant l'influence des dynamiques systémiques à cet égard » (p.183). Toujours selon ces auteurs, ces déficits s'expliqueraient par la non-connaissance de la langue de scolarisation, la défavorisation marquant leur situation familiale ainsi que par différentes pratiques 


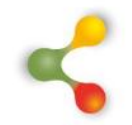

\section{REVUE HYBRIDE DE L'ÉDUCATION}

culturelles. Ainsi, en sous-estimant les ressources particulières des élèves issus de l'immigration ; l'institution scolaire est susceptible de contribuer à ces dynamiques systémiques.

Dès lors, comment amener le personnel à reconnaître la diversité ethnoculturelle, à en identifier la valeur et le potentiel pédagogique pour sortir enfin de la pédagogie compensatoire ? II s'agit probablement d'un échec ou tout de moins d'un enjeu encore présent des pratiques de formation (Lapeyre, 2005 ; Perregaux, 2010). Dans le cas suisse notamment, certaines études montrent les difficultés des instituts de formation des enseignants à préparer efficacement les enseignant-e-s (Sieber et Bischoff, 2007) dont les représentations déficitaires sont robustes (Ramel, 2014). Mis en relation avec les enquêtes récentes sur la présence du racisme à l'école par exemple (Confédération suisse, 2016) ou de la surreprésentation des élèves issus de l'immigration dans l'enseignement spécialisé (Bauer, 2018), force est de constater que la mission de formation du personnel scolaire reste empreinte de défi.

En effet, dans cette contribution nous avons mis en évidence l'inadéquation de l'école à reconnaître les élèves à ressources particulières. Cette expression, qui met l'accent sur le savoir et les potentialités des élèves de l'immigration, interpelle les professionnel-le-s à dépasser leurs seules reconnaissance et valorisation, pour les mobiliser activement dans le cadre scolaire et en particulier face aux apprentissages.

Ainsi, les formations elles-mêmes sont interrogées sur leur capacité à outiller les enseignant-e-s en termes de déconstruction de stéréotypes et de préjugés ainsi que dans la lutte contre le racisme et la discrimination (Gay et Laffranchini Ngoenha, 2018). Les compétences interculturelles et inclusives, bien qu'indispensables dans une société structurellement hétérogène et plurielle, sont souvent sous-représentées dans les curricula de formation (Larochelle-Audet, Borri-Anadon et Potvin, 2016 ; Mc Callister et Irvine, 2002 ; Sieber et Bischoff, 2007).

Par ailleurs, ces actions resteraient superficielles sans le concours de l'institution scolaire dans le changement de paradigme pour la mise en œuvre du projet d'école inclusive. En effet, la notion de besoins éducatifs particuliers de l'école se réfère à la nécessité de s'affranchir de la forme scolaire ségrégative, où, par souci de rationalité et rentabilité, les enfants ont été incorporés dans des classes d'âges et de savoir (Conseil supérieur de l'éducation, 2016). La réussite scolaire basée sur la sélection et la performance n'a fait qu'homogénéiser ultérieurement les classes, la transmission du savoir s'étant structurée principalement autour d'un cadre normatif plutôt que sur les ressources que les élèves apportent à l'enseignement. 


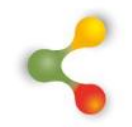

\section{REVUE HYBRIDE DE L'ÉDUCATION}

Ce renversement de perspective engendre aussi la nécessité de considérer, au sein même de la formation, l'influence du cadre institutionnel susceptible de concourir à la non-adhésion ou à l'abandon des professionnel-le-s de l'éducation quant au projet d'école inclusive, et à l'épuisement professionnel. Plusieurs facteurs contribuent en effet à l'absence ou à la faible mobilisation des professionnel-le-s comme la peur de l'inconnu et le manque de ressources personnelles pour faire face aux nouveaux besoins engendrés chez ces derniers, le sentiment d'être peu valorisé ou respecté dans ce nouveau contexte, la crainte de voir sa position professionnelle se modifier et le sentiment d'être dans l'obligation de participer à une action de changement (Albanese et Fiorili, 2009 ; Doudin, Curchod-Ruedi et Baumberger, 2009). En effet, la pédagogie inclusive peut être perçue comme quelque chose de plus, un effort supplémentaire à fournir. Ce contexte a priori négatif lance un défi encore plus grand aux formateurs-trices de futur-e-s enseignant-e-s pour opérer les changements de paradigmes nécessaires à la réalisation de l'école inclusive.

Par conséquent, le projet d'école inclusive nécessite une ouverture et une souplesse dans les modes d'organisation scolaire notamment dans un contexte où l'accent est principalement mis sur la réussite scolaire au sens strict des contenus disciplinaires ; au contraire, elle prend son envol dans un contexte où la socialisation et le sentiment de bien-être sont mis en avant par les professionnel-le-s de l'éducation. Les liens enseignant-e-sélèves et élève-élève, la prise de conscience du potentiel des enfants ou des jeunes, semblent aider les enseignant-e-s tout en renforçant leur sentiment de compétence professionnelle (Ramel et Doudin, 2009).

En conclusion, l'inclusion scolaire, en tant qu'école pour tous, constitue le nouveau défi des systèmes éducatifs. Sa mise en œuvre exige, de la part de tous les acteurs-trices scolaires, un changement de posture professionnelle. Or, l'adaptation de celle-ci à de nouvelles options éducatives ne se décrète pas seulement par une volonté politique ou légale. Elle passe par une prise en compte réelle des besoins liés à cette évolution et qui émergent à différents niveaux : les enseignant-e-s et les intervenant-e-s dans leur pratique, les directions d'établissements dans leur rôle d'encadrement, l'institution scolaire dans sa fonction de garante des conditions de scolarisation. Le succès de la politique inclusive dépendra en effet en grande partie de l'adéquation des réponses apportées aux différents partenaires engagés dans son application, c'est-à-dire des besoins particuliers de l'école elle-même. Ce modèle d'école inclusive serait le point d'alliance de l'éducation interculturelle et de la pédagogie spécialisée. II s'agit à présent pour l'ensemble des professionnel-le-s de l'éducation de reconnaître, valoriser et tirer parti de la diversité afin de saisir la chance que les élèves issus de l'immigration, avec leurs ressources 


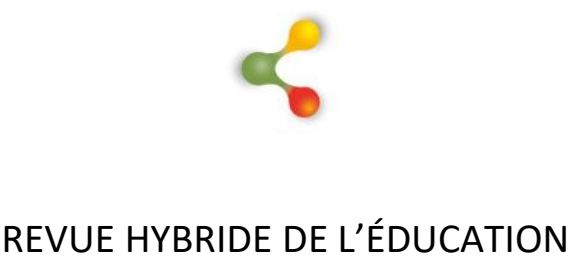

éducatives particulières, donnent à l'école et à la société, afin de rendre la justice sociale effective. 


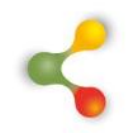

\section{REVUE HYBRIDE DE L'ÉDUCATION}

\section{Références}

Abdallah-Pretceille, M. (1999). L'éducation interculturelle. Paris, France : PUF.

Akkari, A. (2009). Introduction aux approches interculturelles en éducation. Genève, Suisse : Université de Genève.

Akkari, A., Loomis, C. et Bauer, S. (2012). From accommodating to using diversity by teachers in Switzerland. The Journal of Multiculturalism in Education, 7(1), 1-11.

Albanese, O. et Fiorilli, C. (2009). Le risque d'épuisement professionnel des enseignantes et des enseignants de soutien à l'intégration : le cas italien. Revue des HEP et institutions assimilées de Suisse romande et du Tessin, 9, 33-46.

Armand, F. (2016). L'enseignement du français en contexte diversité linguistique au Québec : idéologies linguistiques et exemples de pratiques en salle de classe. Dans M. Potvin, M.-O. Magnan et J. Larochelle-Audet (dir.), La diversité ethnoculturelle, religieuse et linguistique en éducation (pp.172-182). Montréal, Canada : Fides Éducation.

Armand, F., Hardy, M.-H., et Lemay, B. (2014). Le soutien en langue maternelle: une approche novatrice pour les élèves allophones en difficulté ! Québec français, 172, 23-24.

Armand, F. et Maraillet, E. (2013). Éducation interculturelle et diversité linguistique. Repéré à http://www.elodil.umontreal.ca/guides/educationinterculturelle-et-diversite-linguistique/

Artiles, A. et Dyson, A. (2005). Inclusive Education in the Globalization Age : The Promise of Comparative Cultural-Historical Analysis. Dans David Mitchell (dir.), Contextualizing Inclusive Education: Evaluating Old and New International Perspective (pp.37-63). New York, États-Unis : Routledge.

Artiles, A. J., Trent, S. C. et Palmer, J. (2004). Culturally diverse students in special education : Legacies and prospects. Dans J. A. Banks et C. M. Banks (dir.), Handbook of research on multicultural education (pp.716735). San Francisco, CA, États-Unis : Jossey-Bass.

Barral, C. et Roussel, P. (2002). De la ClH [classification international du handicap] à la CIF [classification international du fonctionnement] : le processus de révision. Handicap, revue des sciences humaines et sociales, 94-95, 1-23. 


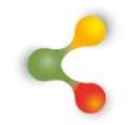

\section{REVUE HYBRIDE DE L'ÉDUCATION}

Bauer, S. (2018). L'enseignement spécialisé est-il (toujours) pertinent pour les élèves issus de la migration? Revue suisse de pédagogie spécialisée, 4, 47-53.

Bauer, S. (2017). Diriger un établissement multiculturel à Genève ou Montréal : le leadership à l'épreuve de la reconnaissance de la diversité. Canadian Journal for New Scholars in Education/ Revue canadienne des jeunes chercheurs et chercheurs en éducation, 8(1), 10-17.

Benoît, H. et Plaisance, E. (2009). L'éducation inclusive en France et dans le monde. La nouvelle revue de l'adaptation et de la scolarisation, Hors Série(5), 3-8.

Borri-Anadon, C. (2014). Pratiques évaluatives des orthophonistes à l'égard des élèves issus de minorités culturelles: une recherche interprétative-critique. Thèse de Doctorat inédite, Faculté des sciences de l'éducation. Université du Québec à Montréal, Montréal, Canada.

Borri-Anadon, C. (2016). Les enjeux de l'évaluation des besoins des élèves en contexte de diversité. Dans M. Potvin, M.-O. Magnan et J. LarochelleAudet (dir.), La diversité ethnoculturelle, religieuse et linguistique en éducation: théorie et pratique (p. 215-224). Anjou, Canada: Fides Éducation.

Borri-Anadon, C., Potvin, M. et Larochelle-Audet, J. (2015). La pédagogie de l'inclusion, une pédagogie de la diversité. Dans N. Rousseau (dir.), La pédagogie de l'inclusion scolaire, un défi ambitieux et stimulant (pp.49-64). Québec, Canada : Presses de l'Université du Québec.

Borri-Anadon, C., Savoie-Zajc, L. et Lebrun, M. (2015). Pratiques évaluatives des orthophonistes scolaires à l'égard des élèves de minorités culturelles : différenciation, uniformisation et normalisation. Recherches \& Éducations, 14, 81-92.

Cartledge, G. (2005). Restrictiveness and Race in Special Education : The Failure to Prevent or to Return. Learning Disabilities : A Contemporary Journal, 3(1), 27-32.

Castellotti, V., Coste, D., et Duverger, J. (2008). Propositions pour une éducation au plurilinguisme en contexte scolaire. Paris, France

: ADEB.

Castellotti, V. et Moore, D. (2002). Représentations sociales des langues et enseignements. Strasbourg, France : Conseil de l'Europe. 


\section{8}

\section{REVUE HYBRIDE DE L'ÉDUCATION}

Confédération suisse (2016). Discrimination raciale en Suisse. Rapport du Service de lutte contre le racisme. Berne, Suisse : Confédération suisse.

Conseil supérieur de l'éducation (2016). Remettre le cap sur l'équité : Rapport sur l'état et les besoins de l'éducation 2014-2016. Québec, Canada : Conseil supérieur de l'éducation, Gouvernement du Québec.

Côté, R. (1998). Apprendre. Formation expérientielle stratégique. Québec, Canada : Presses de l'Université du Québec.

Cummins, J. (2000). Language, power, and pedagogy: Bilingual children in the crossfire. Clevedon, Royaume-Uni : Multilingual Matters.

Cummins, J. (2001). La langue maternelle des enfants bilingues, Qu'est-ce qui est important dans leurs études ? Sprogforum, 19, 15-20.

Doudin, P.-A, Curchod-Ruedi, D., et Baumberger B. (2009). Inclure ou exclure des élèves en difficulté : quelles conséquences pour les enseignantes et les enseignants ? Revue des HEP et institutions assimilées de Suisse romande et du Tessin, 9, 11-32.

Dubet, F. (2009). Les dilemmes de la justice. Dans J. L. Derouet et M.-C. Derouet-Besson (dir.), Repenser la justice dans le domaine de l'éducation et de la formation (pp.29-46). Berne, Suisse : Peter Lang.

Ebersold, S. et Detraux, J.-J. (2013). Scolarisation et besoin éducatif particulier: enjeux conceptuels et méthodologiques d'une approche polycentrée. Alter-European journal of disability research, 7(2), 102-115.

Élodil et ERIT. (2013) Guide Théâtre Pluralité ÉLODIL. Repéré à http://www.elodil.umontreal.ca/fileadmin/documents/Guides/tpe/12complet.pdf

Felouzis, G., Charmillot, S., et Fouquet-Chauprade, B. (2011). Les inégalités scolaires en Suisse et leurs déclinaisons cantonales : l'apport de l'enquête Pisa 2003. Schweizerische Zeitschrift für Soziologie, 37(1).

Fougeyrollas, P. (2002). L'évolution conceptuelle internationale dans le champ du handicap : Enjeux sociopolitiques et contributions québécoises. Perspectives interdisciplinaires sur le travail et la santé, 4 (2).

Gay, D. et Laffranchini Ngoenha, M. (2018). Comment enseigner l'interculturel. Propositions d'enseignements pour déconstruire les stéréotypes. Revues des HEP et institutions assimilées de Suisse romande et du Tessin. Formation et pratiques d'enseignement en question, 23, 177193. 


\section{REVUE HYBRIDE DE L'ÉDUCATION}

Gauthier, R. (2005). Le rapport à l'institution scolaire chez de jeunes amérindiens en fin de formation secondaire: contribution à la compréhension du cheminement scolaire chez les Autochtones. Thèse de doctorat inédite, Sciences de l'Éducation, Université du Québec à Chicoutimi, Canada.

Gremion, M., Noël, I., et Ogay, T. (2013). Éducation interculturelle et pédagogie spécialisée. Tensions et ambiguïtés des discours sur la différence. Schweizerische Zeitschrift für Bildungswissenschaften, 35(1), 53-69.

Hibel, J., et Jasper, A. D. (2012). Delayed Special Education Placement for Learning Disabilities Among Children of Immigrants. Social Forces, 91(2), 503-530.

Hirsch, S. (2018). Prendre en compte la diversité religieuse à l'école québécoise: les défis de formation des futurs enseignants du cours Ethique et culture religieuse. Dans Borri-Anadon, C., Gonçalves, G., Hirsch, S., et di Paula Queiroz Odinino, J. (dir.). La formation des éducateurs en contexte de diversité ethnoculturelle: une perspective comparative Québec-Brésil (pp.142-158). Wisconsin, États-Unis : Deep University Press.

Hirsch, S., Audet, G., et Turcotte, M. (2015). Vivre ensemble. Aborder les sujets sensibles avec les élèves. Guide pédagogique. Centre d'intervention pédagogique en contexte de diversité. Commission scolaire Marguerite Bourgeoys. Repéré à https://www.cipcd.ca/wpcontent/uploads/2014/04/CSMB_-Guide_sujets-sensibles_final.pdf

Lapeyre, M. (2005). L'école inclusive peut-elle réussir là où l'intégration a échoué ? Reliance, 16, 36-42.

Larochelle-Audet, J. et al., 2018. Les compétences des directions en matière d'équité et de diversité : pistes pour les cadres de référence et la formation. Rapport de recherche, Observatoire sur la formation à la diversité et l'équité, UQAM.

Larochelle-Audet, J. Borri-Anadon, C. et Potvin, M., (2016). La formation interculturelle et inclusive des enseignant(e)s: conceptualisation et opérationnalisation de compétences professionnelles, Éducation et francophonie, 44, 172-195.

Lavoie, G., Thomazet, S., Feuilladieu, S., Pelgrims, G. et Ebersold, S. (2013). Construction sociale de la désignation des élèves à 'besoins éducatifs particuliers' : incidences sur leur scolarisation et sur la formation 


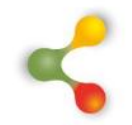

\section{REVUE HYBRIDE DE L'ÉDUCATION}

des enseignants, Alter-European Journal of Disability Research, 7(2), 93101.

Lindsay, G. (2007). Educational psychology and the effectiveness of inclusive education/mainstreaming. British Journal of Educational Psychology, 77(1), 1-24.

Mc Andrew, M., Balde, A., Bakhshaei, M., Tardif-Grenier, K., Audet, G., Armand, F., ...Rousseau, C. (2015). La réussite éducative des élèves issus de l'immigration. Dix ans de recherche et d'intervention au Québec. Montréal, Canada : Presses de l'Université de Montréal.

McAllister, G., et Irvine, J. J. (2000). Cross cultural competency and multicultural teacher education. Review of educational research, 70(1), 324.

Nicollin, L., et Muller Mirza, N. (2013). Le rapport à l'altérité et à la diversité culturelle dans les plans d'étude de Suisse romande : Quelles conceptions d'une éducation à l'altérité ? (Rapport au Fond National Suisse 100013132292). Lausanne, Suisse : Université de Lausanne.

Organisation de coopération et de développement économique [OCDE]. (2016). PISA, 2015 Results : Excellence and Equity in Education. (vol. 1). Paris, France : OCDE.

Organisation de coopération et de développement économiques [OCDE] (2008). Élèves présentant des déficiences, des difficultés et des désavantages sociaux: politiques, statistiques et indicateurs - Édition 2007. Paris, France : OCDE

Pelgrims, G. (2012, septembre). Des élèves déclarés en difficulté aux besoins éducatifs particuliers en passant par l'école inclusive: de quoi parle-t-on ? Conférence présentée au congrès de la Conférence romande et tessinoise des chefs d'établissement secondaire (CROTCES), BienneMarcolin.

Perregaux, Ch. (2010). Education pour l'inclusion : vers une égalité et une différenciation de traitement pour des populations scolaires à besoins spécifiques. Dans C. Acedo, A. Akkari et K. Müller (dir.). L'éducation pour l'inclusion: de la recherche aux réalisations pratiques (pp.37-48). Paris, France : UNESCO.

Plaisance, E. (2000). Les mots de l'éducation spéciale. Dans Chauvière, M. et Plaisance, E. (dir.), L'école face aux handicaps. Éducation spéciale 


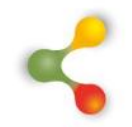

\section{REVUE HYBRIDE DE L'ÉDUCATION}

ou éducation intégrative? (pp.16-29). Paris, France : Presses universitaires de France.

Potvin, M. (2013). L'éducation inclusive et antidiscriminatoire : fondements et perspectives. Dans Mc Andrew, M., Potvin, M. et Borri-Anadon, C. (dir.), Le développement d'institutions inclusives en contexte de diversité (pp.926). Québec, Canada : Presses de l'Université du Québec.

Prud'homme, L., Bergeron, G. et Borri-Anadon, C. (2016). Apprendre à différencier: défis professionnels et pistes d'action pour reconnaître, valoriser et tirer parti de la diversité. Dans M. Potvin, M.-O. Magnan et J. Larochelle-Audet (dir.), La diversité ethnoculturelle, religieuse et linguistique en éducation: théorie et pratique (pp.162-171). Anjou, Canada : Fides Éducation.

Prud'Homme, L., Duchesne, H., Bonvin, P., et Vienneau, R. (2016). L'inclusion scolaire: ses fondements, ses acteurs et ses pratiques. Louvain-la-Neuve, Belgique : De Boeck Supérieur.

Prud'homme, L. et Ramel, S. (2016). Introduction. Dans L. Prud'homme, H. Duchesne, P. Bonvin et R. Vienneau (dir.), L'inclusion scolaire: ses fondements, ses acteurs et ses pratiques (pp.15-17). Louvain-la-Neuve, Belgique : De Boeck Supérieur.

Prud'homme, L., Vienneau, R., Ramel, S. et Rousseau, N. (2011). La légitimité de la diversité en éducation : réflexion sur l'inclusion. Éducation et francophonie, 39(2), 6-22.

Ramel, S. (2014). Elèves en situation de handicap ou ayant des besoins éducatifs particuliers : quelles représentations chez de futurs enseignants? Revue suisse de pédagogie spécialisée, 3, 20-26.

Ramel, S. (2015). D'une rhétorique inclusive à la mise en œuvre de moyens pour la réaliser. Revue suisse de pédagogie spécialisée, 3, 22-28.

Ramel, S. et Doudin, P. (2009). Intégration et inclusion scolaire. Du déclaratif à leur mise en œuvre. Revue des HEP et institutions assimilées de Suisse romande et du Tessin, 9, 5-7.

Ramel, S., et Vienneau, R. (2016). Des fondements sociologiques de l'inclusion scolaire aux injonctions internationales. Dans Prud'Homme, L., Duchesne, H., Bonvin, P., et R. Vienneau (dir.), L'inclusion scolaire : ses fondements, ses acteurs et ses pratiques (pp.25-38.). Louvain-la-Neuve, Belgique : De Boeck Supérieur. 


\section{REVUE HYBRIDE DE L'ÉDUCATION}

Sieber, S. et Bischoff, 2007. Rapport. Examen de la situation actuelle de la pédagogie interculturelle au sein des hautes écoles pédagogiques et des établissements de formation des enseignants de Suisse. Berne, Suisse : Conférence suisse des rectrices et recteurs des hautes école pédagogiques (COHEP).

Terrail, J.-P. (2016). Pour une école de l'exigence intellectuelle. Changer de paradigme pédagogique. Paris, France : La Dispute.

Thomazet, S. (2012). Du handicap aux besoins éducatifs particuliers. Le Français aujourd'hui, 177, 11-17.

UNESCO (1997). International Standard Classification of Education. Paris, France : UNESCO

UNESCO (2009). Les principes directeurs pour l'inclusion dans l'éducation. Paris, France: UNESCO. Repéré à http://www.inclusive-education-inaction.org/iea/dokumente/upload/43806 177849f.pdf

UNESCO (2013). Compétences interculturelles. Cadre conceptuel et opérationnel. Paris, France: UNESCO. Repéré à http://unesdoc.unesco.org/images/0021/002197/219768f.pdf

Waitoller, F. R. ; Artiles, A. J. et Cheney, D. A. (2009). The Miner's Canary : A Review of Overrepresentation Research and Explanations. The Journal of Special Education, 44(1), 29-49. 